\title{
PRINCIPAIS ALTERAÇÕES FISIOLÓGICAS DECORRENTES DA OBESIDADE: UM ESTUDO TEÓRICO
}

MAIN PHYSIOLOGICAL CHANGES DUE TO OBESITY: A THEORETICAL STUDY

PRINCIPALES CAMBIOS FISIOLÓGICOS DERIVADOS DE LA OBESIDAD: UN ESTUDIO TEÓRICO

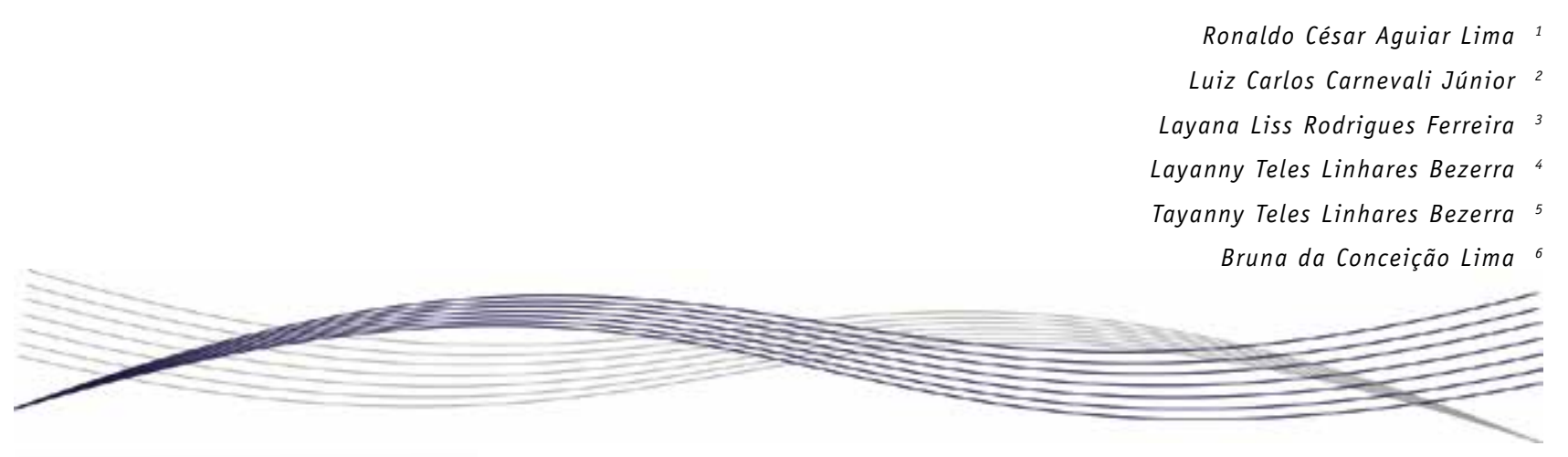

Palavras-chave:

Obesidade; Fisiologia; Adipocinas; Ciências da Saúde.

Keywords:

Obesity; Physiology; Adipokines; Health Sciences.

Palabras clave: Obesidad; Fisiología; Adipoquinas; Ciencias de la Salud.

Submetido: $25 / 06 / 2018$

Aprovado: $22 / 11 / 2018$

Autor(a) para Correspondência: Ronaldo César Aguiar Lima Rua Betânia 10, n. 1612

Cidade Nova II Mucambo-CE 62170-000 E-mail: ronaldocalima@gmail.com

\section{RESUMO}

Este artigo descreve as principais alterações fisiológicas decorrentes da obesidade. Trata-se de uma pesquisa desenvolvida a partir da análise de estudos obtidos nas bases on-line SciELO, MedLine, Lilacs e PubMed. Constatou-se que as grandes vilãs da obesidade são as adipocinas substâncias produzidas pelos adipócitos e capazes de exercer inúmeras funções no organismo. De modo geral, o aumento do tecido adiposo leva a "efeitos de massa", com repercussões na dinâmica ventilatória, por diminuir a complacência pulmonar, o que resulta em pneumopatias restritivas. Além disso, observou-se que níveis aumentados de adipocinas estão diretamente relacionados à expressão de mediadores inflamatórios (TNF- $\alpha, I L-1 \beta$ e IL-6), promovendo um processo inflamatório crônico. Nos músculos, as adipocinas retardam as miocinas (responsáveis pelo turnover muscular), levando a fragilidade muscular e óssea, e competem com a osteocalcina (responsável pelo turnover ósseo), impedindo o remodelamento ósseo. Conclui-se que as adipocinas promovem diversas adaptações sistêmicas, o que demanda novos estudos para o pleno conhecimento e controle da obesidade no campo das ciências da saúde.

\footnotetext{
1. Médico. Especialista em Fisiologia Aplicada às Ciências da Saúde pela Universidade Estácio de Sá (Estácio). Plantonista do Hospital Municipal Senador Carlos Jereissati (HMSCJ). Mucambo (CE), Brasil. E-mail: ronaldocalima@gmail.com

2. Educador Físico. Doutor em Biologia Celular e Molecular pela Universidade de São Paulo (USP). Coordenador do curso de especialização em Fisiologia Aplicado as Ciências da Saúde, pela Universidade Estácio de Sá, São Paulo (SP), Brasil.

3. Enfermeira graduada pela Universidade Estadual Vale do Acaraú (UVA). Diretora de Enfermagem da RL Clínica Integrada. Mucambo (CE), Brasil.

4. Enfermeira graduada pelo Instituto Superior de Teologia Aplicada (Inta). Enfermeira no Serviço de Oncologia da Santa Casa de Misericórdia de Sobral (SCMS). Sobral (CE), Brasil.

5. Enfermeira graduada pelo Inta. Enfermeira plantonista do HMSCJ. Mucambo (CE), Brasil.

6. Enfermeira graduada pelo Inta. Coordenadora de Enfermagem do HMSCJ. Mucambo (CE), Brasil.
} 


\section{ABSTRACT}

This article describes the main physiological changes due to obesity. This is a research developed through analysis of studies obtained in the online bases SCIELO, MedLine, LILACS, and PubMed. It has been found that the great villains of obesity are adipokines - substances produced by adipocytes and capable of exerting numerous functions in the body. In general, increased adipose tissue leads to 'mass effects,' with repercussions on ventilatory dynamics, as lung compliance decreases, resulting in restrictive lung diseases. Also, increased adipokine levels have been shown as directly related to expression of inflammatory mediators (TNF- $\alpha, I L-1 \beta$ e IL-6), promoting a chronic inflammatory process. In muscles, adipokines slow down myocins (responsible for muscle turnover), leading to muscle and bone weakness, and they compete with osteocalcin (responsible for bone turnover), preventing bone remodeling. It is concluded that adipokines promote several systemic adaptations, which require new studies to achieve full knowledge on and control of obesity in the field of health sciences.

\section{RESUMEN}

Este artículo describe los principales cambios fisiológicos derivados de la obesidad. Esta es una investigación desarrollada a través del análisis de estudios obtenidos en las bases en línea SciELO, MedLine, LILACS y PubMed. Se ha encontrado que las grandes villanas de la obesidad son las adipoquinas - sustancias producidas por los adipocitos y capaces de ejercer numerosas funciones en el cuerpo. En general, el aumento de tejido adiposo conduce a "efectos de masa", con repercusiones en la dinámica ventilatoria, a medida que disminuye el cumplimiento pulmonar, lo que resulta en enfermedades pulmonares restrictivas. Además, se ha demostrado que el aumento de los niveles de adipoquinas está directamente relacionado con la expresión de mediadores inflamatorios (TNF- $\alpha$, $I L-1 \beta$ e IL-6), lo que promueve un proceso inflamatorio crónico. En los músculos, las adipoquinas retrasan las miocinas (responsables del turnover muscular), lo que lleva a la debilidad muscular y ósea, y compiten con la osteocalcina (responsable del turnover óseo), evitando la remodelación ósea. Se concluye que las adipoquinas promueven varias adaptaciones sistémicas, lo que requiere nuevos estudios para lograr conocimiento completo y control de la obesidad en el campo de las ciencias de la salud.

\section{INTRODUÇÃO}

A obesidade é definida pelo acúmulo de tecido adiposo no organismo, resultante da quebra do balanço energético, também podendo ser causada por doenças genéticas e/ou endócrino-metabólicas ${ }^{1}$. A Organização Mundial da Saúde (OMS) conceitua a obesidade como doença crônica, de difícil etiologia e de controle complexo, considerando-a como um transtorno alimentar da modernidade.

A Sociedade Brasileira de Endocrinologia e Metabologia (SBEM) alerta que a obesidade vai muito além do acúmulo excessivo de gordura, pois está associada a diversas desordens na fisiologia endócrino-metabólica, propiciando um estado que predispõe a endocrinometabolopatias ${ }^{2}$.

Já o Ministério da Saúde afirma que a obesidade, mesmo sendo de difícil definição, constitui um problema de saúde pública, pois já acomete cerca de $10 \%$ da população brasileira, sendo que outros $40 \%$ já apresentam sobrepeso - ou seja, quase metade dos brasileiros rumam para a obesidade ${ }^{3}$.

Assim, pela complexidade, o aumento do peso corporal só poderá ser compreendido em sua multifatorialidade, pois envolve desde fatores biológicos até aspectos históricos, interpolando-os com características ecológicas, econômicas, sociais, culturais e políticas ${ }^{2-3}$.

Desse modo, percebe-se que a obesidade é um fenômeno complexo que envolve muito mais do que o aumento do peso. É o acúmulo de tecido adiposo, que desempenha diversas funções ainda não plenamente compreendidas no organismo $0^{4}$. Tal acúmulo de gordura acarreta tanto o aumento do volume corporal como uma exacerbação das funções do tecido em questão, o que configura o principal fator responsável pelas desordens fisiológicas decorrentes da obesidade ${ }^{2,4}$.

No tocante as desordens fisiológicas desencadeadas pela obesidade, aquelas que acometem o sistema endócrino-metabólico (obesidade mórbida, diabetes mellitus tipo 2), bem como as que agridem o sistema cardiovascular (acidente vascular encefálico - AVE - e infarto agudo do miocárdio - IAM), são as mais conhecidas ${ }^{2-4}$.

No entanto, as alterações fisiológicas promovidas pelo excesso de adipócitos vão além dos distúrbios metabólicos e cardiovasculares e acometem outros sistemas, como o nervoso, o cardiorrespiratório, o imunológico etc. ${ }^{5}$. Com diversas outras alterações relacionando-se ao excesso de gordura e seus 
biomarcadores, como osteoartrite, distúrbios do humor e do sono, alterações menstruais e, inclusive, alguns tipos de câncer ${ }^{2,5-6}$.

Por tantos motivos, a importância da obesidade aumentou bastante nas últimas décadas, levando a OMS a classificá-la com uma pandemia e a ressaltar que os danos à saúde e o número de óbitos relacionados ao excesso de gordura já ultrapassam os relativos à desnutrição $0^{7}$. Por outro lado, acompanhando a expansão da obesidade, há uma vasta produção científica sobre o acúmulo de tecido adiposo, com muitos estudos sobre as consequências do excesso de gordura no organismo a ser estudada.

Com base nessa problemática, o estudo teve por objetivo promover uma reflexão teórica sobre a obesidade e os prejuízos do acúmulo de adipócitos no organismo, descrevendo as modificações desde a concepção até a terceira idade. Este artigo descreve as principais alterações fisiológicas decorrentes da obesidade, apontando os mecanismos envolvidos nas diversas desordens promovidas pelo excesso de tecido adiposo e suas substâncias (as adipocinas).

\section{METODOLOGIA}

Trata-se de reflexão teórica sobre a obesidade que busca compreender como o tecido adiposo e seus produtos de sintese (as adipocinas) se relacionam para promover alterações fisiológicas em obesos.

A pesquisa se desenvolveu a partir da análise de estudos obtidos nas bases on-line SciELO, MedLine, Lilacs e PubMed (publicados em português, inglês ou espanhol). Os descritores adotados para busca e seleção dos artigos foram:
- "Obesidade";

- "Adipocinas";

- "Fisiologia";

- "Adaptações";

- "Organismo";

- "Sistemas orgânicos"; e

- "Gordura".

Esses descritores foram inseridos no menu "pesquisa" das bases on-line, com uso do operador booleano "AND": "obesidade" foi o primeiro em todas as buscas, relacionado aos demais.

Para os termos "obesidade" AND "adipocinas" foram obtidos 798 estudos (14 na SciELO; 511 na MedLine; 61 na Lilacs; e 212 na PubMed). 0s artigos tratavam de diversos temas relacionados à obesidade, muitos deles com mais de uma década publicação. Ao acrescentar os demais termos a "obesidade", a quantidade de estudos diminui. Aplicando os critérios "ano de publicação" (com vistas a selecionar artigos com menos de dez anos - de 2009 a 2017) e "texto integral" (excluindo resumos), 21 artigos foram selecionados para prover o embasamento científico deste artigo.

\section{RESULTADOS E DISCUSSÃO}

A produção científica sobre a obesidade é vasta e variada, mas os mecanismos metabólicos que se desenrolam em organismos obesos ainda são pouco esclarecidos, de modo que a amostra deste artigo se limitou a 21 estudos disponiveis nas principais bases de periódicos científicos (Quadro 1).

Quadro 1 - Distribuição dos estudos incluídos na amostra, publicados no período de 2009 a 2017.

\begin{tabular}{|c|c|c|c|}
\hline $\mathbf{N}$ & Titulo & Periódico & Ano \\
\hline 01 & $\begin{array}{l}\text { Anatomical connections between medial and lateral regions of the } \\
\text { hypothalamus concerned with food intake }{ }^{10}\end{array}$ & Science & 2010 \\
\hline 02 & Obesidade infantil e suas relações com o equilíbrio corporal ${ }^{1}$ & Acta Fisiátrica & 2009 \\
\hline 03 & Alterações ortopédicas em crianças e adolescentes obesos ${ }^{28}$ & Fisioter Mov & 2010 \\
\hline 04 & Obesity and asthma: association or coincidence? ${ }^{27}$ & J Pediatr (Rio J) & 2010 \\
\hline 05 & Fatores associados ao metabolismo energético na obesidade ${ }^{16}$ & Nutrire & 2010 \\
\hline 06 & Altered hypothalamic function in dietinduced obesity ${ }^{22}$ & Int $\mathrm{J}$ Obes & 2011 \\
\hline 07 & $\begin{array}{l}\text { Prevalência de alterações metabólicas em crianças e adolescentes } \\
\text { com sobrepeso e obesidade: uma revisão sistemática }{ }^{11}\end{array}$ & Rev Paul Pediatr & 2011 \\
\hline 08 & Impulsividade na obesidade: questões conceituais e metodológicas ${ }^{4}$ & Arq Bras Psicol & 2011 \\
\hline 09 & Compulsão alimentar, obesidade e emagrecimento ${ }^{19}$ & $\begin{array}{c}\text { Revista Brasileira de } \\
\text { Obesidade, Nutrição e } \\
\text { Emagrecimento }\end{array}$ & 2011 \\
\hline
\end{tabular}




\begin{tabular}{|c|c|c|c|}
\hline $\mathbf{N}$ & Titulo & Periódico & Ano \\
\hline 10 & Obesidade: controle neural e hormonal do comportamento alimentar ${ }^{5}$ & Rev Ciênc Méd Biol & 2011 \\
\hline 11 & $\begin{array}{l}\text { Obesidade e estresse entre trabalhadores de diversos setores de } \\
\text { produção: uma revisão integrativa }{ }^{30}\end{array}$ & Acta Paul Enferm & 2011 \\
\hline 12 & Adiponectina e baixo risco cardiometabólico em obesas ${ }^{23}$ & $\begin{array}{l}\text { Arq Bras Endocrinol } \\
\qquad \text { Metab }\end{array}$ & 2011 \\
\hline 13 & $\begin{array}{l}\text { Aterosclerose subclínica e marcadores inflamatórios em crianças e } \\
\text { adolescentes obesos e não obesos }{ }^{25}\end{array}$ & Rev Bras Epidemiol & 2012 \\
\hline 14 & $\begin{array}{l}\text { Relação entre aterosclerose subclínica, pressão arterial e perfil } \\
\text { lipídico em crianças e adolescentes obesos: uma revisão sistemática }{ }^{26}\end{array}$ & $\begin{array}{l}\text { Arq Bras Endocrinol } \\
\text { Metab }\end{array}$ & 2013 \\
\hline 15 & $\begin{array}{l}\text { Peroxidação lipídica e obesidade: Métodos para aferição do estresse } \\
\text { oxidativo em obesos }{ }^{15}\end{array}$ & GE, J Port Gastrenterol & 2013 \\
\hline 16 & $\begin{array}{l}\text { The influence of leptin on Th1/Th2 balance in obese children with } \\
\text { asthma }{ }^{18}\end{array}$ & J Bras Pneumol & 2013 \\
\hline 17 & $\begin{array}{l}\text { Resistência à insulina associada à obesidade: efeitos anti- } \\
\text { inflamatórios do exercício físico }{ }^{20}\end{array}$ & Rev Bras Ciênc Mov & 2014 \\
\hline 18 & Mobile health e excesso de peso: uma revisão sistemática ${ }^{8}$ & $\begin{array}{l}\text { Rev Panam Salud } \\
\text { Pública }\end{array}$ & 2014 \\
\hline 19 & $\begin{array}{l}\text { As multifacetas do excesso de peso na criança: uma revisão } \\
\text { sistemática }{ }^{12}\end{array}$ & $\begin{array}{l}\text { Revista de Atenção à } \\
\text { Saúde }\end{array}$ & 2015 \\
\hline 20 & $\begin{array}{l}\text { A obesidade é um determinante da resistência à insulina mais } \\
\text { importante do que os níveis circulantes de citocinas pró-inflamatórias } \\
\text { em pacientes com artrite reumatoide }{ }^{21}\end{array}$ & Rev Bras Reumatol & 2017 \\
\hline 21 & Obesidade e infertilidade: uma revisão sistemática da literatura9 ${ }^{9}$ & $\begin{array}{l}\text { Revista Interdisciplinar } \\
\text { da UNINOVAFAPI }\end{array}$ & 2017 \\
\hline
\end{tabular}

Fonte: Elaborado pelos autores.

A literatura científica é categórica ao taxar a obesidade como uma complexa pandemia (com mais de 2,1 bilhões de obesos no mundo), resultante da interação de genes, ambiente, estilos de vida e fatores emocionais ${ }^{1,8}$. Desse modo, de 95 a $99 \%$ dos casos não se estabelecem a causa exata da obesidade (obesidade primária) e apenas de 1 a $5 \%$ dos casos atribuem uma causa (obesidade secundária) a endocrinopatias, metabolopatias, drogas etc. ${ }^{8}$.

$0 \mathrm{~s}$ mais recentes estudos científicos alertam que a obesidade - também conhecida como nediez ou pimelose -, embora simplificadamente definida como excesso de gordura corporal, deve ser entendida como uma condição patológica acompanhada por acúmulo de alterações adaptativas desencadeadoras de comorbidades potencialmente fatais ${ }^{3,8}$.

Embora ainda pouco esclarecidos, os estudos revelam que os efeitos das substâncias relacionadas ao tecido adiposo (as adipocinas) são mediadores de "reações" que promovem adaptações deletérias desde a concepção, pois relacionam-se à infertilidade do casal e desencadeiam alterações genéticas com maior risco de malformações em filhos de obesos ${ }^{8-9}$.
$\mathrm{Na}$ infância, 0 excesso de adipocinas age retardando progressivamente o desenvolvimento neuropsicomotor (DNPM) e cognitivo da criança, pois a multiplicação dos adipócitos é mais intensa nos primeiros anos de vida ${ }^{10-11}$, levando a atraso global do desenvolvimento neurológico com repercussões no sistema imunológico, o que deixa a criança obesa mais suscetivel a doenças mentais e infectocontagiosas ${ }^{1,9,11}$ e aumentam as chances de obesidade futura.

A obesidade futura, referida por alguns autores, baseia-se na constatação de que, o tecido adiposo "armazenado" na infância é capaz de multiplicar-se também na vida adulta a partir de novas células, os pré-adipócitos, tornando a obesidade infantil um forte desencadeador de obesidade entre os adultos ${ }^{1,4}$.

$\mathrm{Na}$ adolescência, níveis elevados de adipocinas interferem diretamente no início e na estruturação da puberdade, pois competem com mediadores da sintese de hormônios esteroidais e interferem na expressão e/ou transporte de outros hormônios secundários e na consequente definição dos caracteres sexuais secundários ${ }^{11-12}$. 
Na vida adulta, o excesso de gordura que infiltra os tecidos e órgãos promove lenta substituição lipofílica, com efeitos compressivos, que sabidamente se relacionam à diminuição da qualidade de vida, pois interferem em aspectos psicológicos, aumentado os riscos de transtornos depressivos e multiplicando as chances de desenvolvimento precoce de outros transtornos emocionais bastante comuns entre os obesos ${ }^{13}$.

Já na terceira idade, devido à diminuição do turnover fisiológico, a constituição morfológica é lentamente substituída por tecido adiposo, tornando a "adipolização" um fenômeno inerente ao idoso - ou seja, a lenta e progressiva substituição de estruturas diversas por adipócitos faz parte do envelhecimento ${ }^{14}$.

Assim, os danos promovidos pelo excesso de gordura no organismo idoso consistem na somatória de comorbidades manifestadas na terceira idade ${ }^{14}$, pois o desgaste natural é apenas intensificado pelas adipocinas - classificadas como potencializadoras de comorbidades ${ }^{6,13-14}$.

Todas essas modificações observadas na obesidade são atribuídas às adipocinas, peptídeos que atuam de forma direta na gênese do acúmulo de tecido adiposo, alertando que o tecido adiposo, antes considerado um tecido inerte, demonstra ser um tecido dinâmico $0^{5,15}$.

As adipocinas (leptina, resistina, adiponectina, apelina, vaspina, omentina, proteína estimuladora de acilação etc.) são enfatizadas pelos estudos como as verdadeiras "vilãs" da obesidade ${ }^{15}$, capazes de desenvolver diversos mecanismos e estimular outras tantas vias metabólicas (Quadro 2).

Quadro 2 - Descrição das principais adipocinas, locais de síntese e principal função.

\begin{tabular}{|c|c|c|}
\hline Adipocina & Local de síntese & Função \\
\hline Adiponectina & Adipócitos & $\begin{array}{l}\text { Efeitos contrários às demais adipocinas, relaciona-se à melhora da } \\
\text { sensibilidade à ação da insulina (efeito antagônico em relação ao } \\
\text { hormônio resistina), aumento da atividade da proteína cinase (AMPK) } \\
\text { e aumento da oxidação de ácidos graxos. }\end{array}$ \\
\hline Apelina & Adipócitos & $\begin{array}{l}\text { Proliferação das células endoteliais, seja no estado fisiológico ou } \\
\text { patológico. }\end{array}$ \\
\hline Grelina & Estômago & $\begin{array}{l}\text { Regulador, de curto prazo, da saciedade: aumenta a ingesta e reduz o } \\
\text { catabolismo de gorduras e o gasto energético. }\end{array}$ \\
\hline Leptina & Adipócitos & $\begin{array}{l}\text { Regulador, de longo prazo, da saciedade: Diminui a síntese hipotalâmica } \\
\text { de orexígenos, aumenta a atividade simpática, o metabolismo, o gasto } \\
\text { energético e a produção de hormônio liberador da ACTH. Diminui } \\
\text { produção de insulina. }\end{array}$ \\
\hline Omentina & Adipócitos & Melhora a sensibilidade insulínica. \\
\hline Proteína $G$ & Adipócitos & $\begin{array}{l}\text { Diminui a concentração de ácidos graxos livres no lúmen do capilar, } \\
\text { aumentando a síntese de triacilglicerol pelo tecido adiposo. }\end{array}$ \\
\hline Resistina & Adipócitos & $\begin{array}{l}\text { Associada à resistência insulínica (Hiperinsulinemia), proporciona maior } \\
\text { síntese de marcadores inflamatórios, como TNF- } \alpha \text {, IL-1 e IL- } 6 \text {. }\end{array}$ \\
\hline Vaspina & Adipócitos & $\begin{array}{c}\text { Melhora a sensibilidade à insulina e promove redução da expressão e dos } \\
\text { níveis plasmáticos da insulina. }\end{array}$ \\
\hline Visfatina & Adipócitos & $\begin{array}{c}\text { Atua como fator de estimulação de colônias de células pré- } \beta \text { (PBEF), } \\
\text { tem ação insulinomimética e capacidade de minimizar a resistência } \\
\text { insulínica. }\end{array}$ \\
\hline
\end{tabular}

Fonte: Elaborado pelos autores.

Como se observa no Quadro 2, as substâncias sintetizadas pelos adipócitos são bastante diversificadas, atuando em alças metabólicas complexas e desenvolvendo ações fisiológicas necessárias ao balanço energético ${ }^{16}$. Entretanto, em excesso, essas substâncias promovem desarmonia da homeostase, desencadeando um processo de inflamação contínuo e controlado ${ }^{15}$, apontado como principal fator danoso da obesidade, pois o processo inflamatório permanente, característico da obesidade, que tem como fator causal os elevados níveis de adipocinas, interfere em todos os aparelhos do organismo ${ }^{4}$. 
Como destacado em todos os estudos analisados, qualquer sistema orgânico pode sofrer influência das adipocinas - estas podem sinergir ou antagonizar as ações fisiológicas nos tecidos ${ }^{16}$ ou até competir com outras substâncias, potencializando a ação de outras adipocinas ${ }^{15-16}$.

No contexto da obesidade, algumas adipocinas têm seus mecanismos de ação desajustados, como a grelina, sintetizada no estômago e diretamente relacionada à ingestão alimentar, pois atua regulando o apetite no centro de fome, tem seus mecanismos potencializados mostrando-se ainda mais capaz de sensibilizar as vias de apetite e de estimular o consumo alimentar ${ }^{17}$.

Antagonista natural da grelina, a leptina, a adipocina de maior atividade conhecida no sistema nervoso ${ }^{18}$, é conhecida como controladora da fome, pois atua no hipotálamo, ligando-se a receptores " $0 b^{\prime}$ " (ObR ou LepR) e ativando a via citosólica mediada por JAK2 (Janus Cinase-2), o que estimula a saciedade ${ }^{19}$. A leptina também ativa STAT3 (transdutor de sinal e ativador de transcrição 3 ), que regula a expressão da SOCS-3 (supressor de sinalização de citocinas 3) e da PTP1B (proteína tirosina fosfatase-1B), reforçando a saciedade e, desse modo, controlando o consumo energético-calórico ${ }^{17,19}$ que é vigorosamente estimulado pela grelina ${ }^{17}$.

No entanto, o obeso cursa com altas concentrações de grelina, e para antagonizar os efeitos das elevadas concentrações de grelina, típicas do obeso, a leptina, ao ativar as várias vias metabólicas descritas acima, gera um misto de radicais livres, produtos intermediários das múltiplas vias energéticas estimuladas, que progressivamente levam à oxidação do tecido nervoso no hipotálamo $0^{10,17-18}$, desencadeando um processo inflamatório hipotalâmico irreversível com perda do controle dos mecanismos de fome e saciedade ${ }^{10,18}$ promovendo ainda mais acúmulo de tecido adiposo.

Complicando-se os efeitos danosos do excesso de leptina ao sistema nervoso, surge a compreensão do sinergismo da leptina com a insulina, sendo conhecido que a insulina promove maior expressão dos receptores de leptina ${ }^{20}$. Como a obesidade cursa com um estado de hiperinsulinemia ${ }^{21}$, essa alça de retroalimentação positiva promove cada vez mais oxidação do tecido nervoso, que desencadeia maiores alterações inflamatórias irreversíveis ${ }^{10,18,20-21}$.

Nesse cenário de oxidação do tecido nervoso, outra adipocina assume destaque, a proteína estimuladora de acilação, que, embora não tenha

\section{...a leptina \\ desencadeia \\ calcificação \\ arterial \\ coronariana...}

suas ações totalmente definidas, em ambientes hiperlipídicos, mostra-se capaz de ativar a via da ativação do Toll-like receptor 4 (TLR4), ativando mecanismos de apoptose de neurônios hipocampais e causando danos às áreas neurocognitivas ${ }^{10,22}$, o que cursa com maiores riscos de desenvolver demência precoce $^{6,13}$.

Além dos efeitos cognitivos, os estados hiperlipídicos da obesidade também promovem alterações hemodinâmicas ao hiperestimular os sistemas simpático e renina-angiotensinaaldosterona, levando a maior retenção de sódio e, consequentemente, de líquido - com expressiva sobrecarga do sistema cardiovascular ${ }^{23}$. Manifestando-se com aumento do débito cardíaco associado a aumento da resistência vascular periférica, levando ao desenvolvimento de hipertensão arterial sistêmica (HAS) precoce, que se complica com a forma excêntrica/concêntrica de hipertrofia ventricular esquerda, predispondo a maior risco de arritmia cardíaca e de insuficiência cardíaca congestiva ${ }^{23-24}$.

Ainda em relação à hemodinâmica, descreveuse que a leptina desencadeia calcificação arterial coronariana, justificando o estado de próaterosclerose comum nos obesos ${ }^{18,25}$, e que a resistina aumenta a expressão de moléculas de adesão intercelular-1 e antivascular-1, em células endoteliais, intensificando a gênese aterogênica, além de aumentar a atividade do fator NFkB - sinalizador da indução de adesão dessas moléculas ${ }^{24,26}$.

Tais alterações na dinâmica circulatória promovem disfunção vascular, que, consequentemente, aumenta os riscos cardiovasculares (AVE e IAM) e tantos outros distúrbios cardiovasculares tão recorrentes na população conteporânea $3,23,25-26$, além de prejudicar 0 sistema respiratório, uma vez que mantém estreita relação com a hemodinâmica.

Em termos ventilatórios, pouco se sabe sobre 
as vias que os mediadores da obesidade utilizam para prejudicar a dinâmica respiratória, mas alguns estudos afirmam que a infiltração das estruturas ventilatórias por tecido adiposo diminui a complacência torácica, refletindo em pneumopatias restritivas e pneumopatias inflamatórias ${ }^{27}$.

Reforçando as possíveis alterações na dinâmica ventilatória, há estudos em andamento que apontam uma possivel hiperexcitação do epitélio respiratório pela resistina ${ }^{6,27}$. Tal hiperexcitação desencadearia um epitélio hiper-responsivo típico da doença asmátca ${ }^{27}$, prejudicando as trocas gasosas, levando a falhas na hematose e, consequentemente, fragilizando todos os tecidos que necessitam de oxidação energética, como os componentes do sistema musculoesquelético.

Sobre o musculoesquelético, os estudos descrevem alterações osteomusculares decorrentes da obesidade se devem principalmente às contínuas e altas pressões exercidas nas articulações, desgastando os ossos até torná-los frágeis e quebradiços e levando a um quadro de deformidades e fraturas ósseas ${ }^{28}$.

No entanto, também já se conhecem algumas adipocinas, como a adiponectina e a própria leptina, que podem exercer funções no remodelamento ósse $0^{18,28}$. Com estudos recentes destacando que os mesmos genes que comandam a sintese de leptina competem com os genes que sintetizam osteocalcina, uma molécula sintetizada pelos osteoblastos e considerada o hormônio da remodelação óssea ${ }^{29}$, levando à conclusão de que o aumento da leptina, típica de obesos, envolve diminuição da osteocalcina, com consequente perda do remodelamento ósseo.

Já nos músculos se observa algo semelhante, pois as adipocinas antagonizam as miocinas (substâncias produzidas em resposta à contração muscular que medeiam alguns dos benefícios da atividade física $)^{19}$, levando a progressiva diminuição dos receptores GLUT IV, os principais receptores insulínicos dos músculos, o que gera diminuição da atividade muscular e intensifica o típico estado de hiperinsulinemia dos obesos ${ }^{18,20}$.

Sistemicamente, as repercussões da obesidade também se destacam, pois a principal característica da obesidade (estado de inflamação crônica) se deve a hiperestimulação de mediadores inflamatórios, como Il-6, e expressão de fator de necrose tumoral alfa (TNF- $\alpha)$, com exacerbação dos estímulos próinflamatórios, mas sem efetivamente exercer uma inflamação característica ${ }^{20,28}$.

0 s estudos são unânimes em afirmar que o

\section{...são tantas as modificações fisiológicas promovidas pela obesidade...}

permanente estado de inflamação da obesidade é ocasionado por adipocinas que hiperestimulam proteínas cinase sensíveis a inflamação, como a cinase c-Jun $\mathrm{N}$-teminal (JNK) e a cinase do inibidor do NF-KB (IKK), com ativação contínua da proteína IKK, que fosforila a IKB, proteína que sequestra o NFKB (fator nuclear-KB que transloca ao núcleo e leva à transcrição de genes inflamatórios) no citoplasma, perpetuando uma leve inflamação crônica ${ }^{15,30}$.

Como a leptina é a adipocina mais estudada, sabese que ela induz maior produção de IL-6 e proteína $C$ reativa ( $P C R)$, sem desencadear toda a cascata de inflamação, gerando um estado inflamatório crônico considerado de baixo grau, mas que contribui para o aumento do risco de diabetes mellitus, doenças cardiovasculares dentre outras; embora a magnitude de tal inflamação seja baixa, a cronicidade desse baixo grau inflamatório pode ser decisiva para a progressão da obesidade e de suas comorbidades ${ }^{15}$.

Ainda sobre o permanente estado inflamatório dos obesos, também se conhece que a resistina está relacionada à síntese de marcadores inflamatórios, como TNF- $\alpha$, IL-1 e IL-6, lipoproteína e fosfolipase A2, que, por sua vez, atuam na regulação da síntese de resistina e constituem poderosos inibidores da secreção e expressão de adiponectina ${ }^{15,18,20}$.

Portanto, são múltiplas as ações próinflamatórias das adipocinas. As evidências apontam que o excesso dessas substâncias é tipicamente próinflamatório, sendo as interleucinas IL-1, IL-6 e IL-8, - TNF $\alpha$ e aquelas produzidas por células Th1 (IL-2 e interferon- $\gamma$ ) as mais relacionadas à obesidade ${ }^{15,19,21}$. E tantas alterações nos mecanismos fisiológicos representam importantes fatores de risco para a homeostasia e a harmonia dos sistemas orgânicos.

Desta forma, são tantas as modificações fisiológicas promovidas pela obesidade, que através de conhecimentos mais profundos sobre a dinâmicas adipocinas, vem-se progressivamente entendendo melhor os efeitos danosos da obesidade e a partir 
desses conhecimentos, poder-se-á elaborar medidas eficazes para controle desses efeitos deletérios e potencialmente prejudiciais a vida humana.

\section{CONCLUSÃO}

A obesidade constitui um problema de saúde pública global e os estudos mais recentes apontam significativos percentuais da população vivendo com sobrepeso e/ou obesidade. Diferente dos conceitos de outrora, que relacionavam obesidade a saúde ou a melhores condições de vida, hoje, o excesso de tecido adiposo é analisado de modo mais profundo, pois se vêm desvendando lentamente os reais riscos do acúmulo de tecido adiposo.

0 crescente interesse no tema obesidade tem estimulado a elaboração de um número cada vez maior de estudos no campo da fisiologia humana, esclarecendo os mecanismos bioquímicos nos quais o tecido adiposo interfere.

Desse modo, a obesidade passou a ser muito mais do que o simples acúmulo de gordura corporal, pois a comunidade científica já sabe que a obesidade representa o acúmulo de um tecido vivo (tecido adiposo), dinâmico e capaz de secretar substâncias químicas (adipocinas) que podem atuar em diversos sítios e interferir em toda a fisiologia do organismo.

Interferindo em órgãos e sistemas, os produtos do tecido adiposo ativam processos inflamatórios silenciosos, mas com resultados devastadores para a homeostasia do organismo. Nesse contexto, os recentes estudos sobre a fisiologia da obesidade surgem como promissores, uma vez que, com o conhecimento sobre como o tecido adiposo se comporta, bem acerca de como seus produtos de secreção agem nos sistemas humanos, pode-se manejar melhor esse problema de saúde que vem trazendo tantos agravos à humanidade.

A partir do conhecimento detalhado da fisiologia da obesidade, pode-se adotar melhores métodos preventivos, diminuindo a incidência de obesidade; ademais, novas abordagens terapêuticas podem

$$
\begin{gathered}
\text {...a obesidade } \\
\text { constitui um } \\
\text { problema de } \\
\text { saúde pública } \\
\text { global. }
\end{gathered}
$$

ser instituídas, tornando as atuais terapias menos complexas.

Portanto, o conhecimento da fisiologia da obesidade se mostra importante não só para os profissionais da saúde, mas para toda a população. 0 pleno conhecimento e controle da obesidade demanda novos estudos no campo das ciências da saúde, com vistas a combater esse problema de saúde pública que acomete tantos indivíduos no mundo atual.

\section{CONTRIBUIÇÃO DOS AUTORES}

Ronaldo César Aguiar Lima e Luiz Carlos Carnevali Júnior contribuíram com a realização da pesquisa, o delineamento do estudo e a redação e revisão crítica do manuscrito. Layana Liss Rodrigues Ferreira contribuiu com a realização da pesquisa e a redação e revisão crítica do manuscrito. Layanny Teles Linhares Bezerra, Tayanny Teles Linhares Bezerra e Bruna da Conceição Lima contribuíram com a realização da pesquisa e a redação do manuscrito.

\section{REFERÊNCIAS}

1. Lemos LFC, David AC, Teixeira CS, Mota CB. obesidade infantil e suas relações com o equilíbrio corporal. Acta Fisiátrica [serial on the internet]. 2009 [cited 2018 Nov 28];16(3):138-41. Available from: http://www.revistas.usp.br/actafisiatrica/ article/view/103218/101635

2. Sociedade Brasileira de Endocrinologia e Metabologia. Obesidade: introdução [document on the internet]. 2010 [cited 2014 Nov 22]. Available from: https://www.endocrino.org.br/obesidadeintroducao

3. Brasil. Obesidade. Brasília (DF): Ministério da Saúde; 2006. (Cadernos de Atenção Básica, n. 12).

4. Pereira C, Chehter EZ. Impulsividade na obesidade: questões conceituais e metodológicas. Arq Bras Psicol [serial on the internet]. 2011 [cited 2018 Nov 28];63(1):1-110. Available from: http:// pepsic.bvsalud.org/pdf/arbp/v63n1/v63n1a06.pdf

5. Landeiro FM, Quarantini LC. Obesidade: controle neural e hormonal do comportamento alimentar. Rev Ciênc Méd Biol [serial on the internet]. 2011 [cited 2018 Nov 28];10(3):236-45. Available from: http://professoralexandrerocha.com.br/wp-content/ uploads/2017/05/0besidade-controle-neural-ehormonal-do-comportamento-alimentar.pdf

6. Lima RCA, Ferreira LLR, Lima ECA, Lima CB, Bezerra LTL, Vasconcelos AMB, et al. Associação de obesidade com o câncer: uma reflexão teórica. I Congresso Internacional de Saúde Pública do Delta do Parnaíba; 2015; Parnaíba-PI. Anais. (Sanare (Sobral). 2015;15(Spec).

SANARE, Sobral - v.17 n.02, p.56-65, Jul./Dez. - 2018 
7. Organização Mundial da Saúde. OMS alerta que obesidade está aumentando em vários países. [document on the internet], 2015 [cited 2015 Mar 11]. Available from: https://news.un.org

8. Sarno F, Canella DS, Bandoni DH. Mobile health e excesso de peso: uma revisão sistemática. Rev Panam Salud Pública [serial on the internet]. 2014 [cited 2018 Nov 28];35(5/6):424-31. Available from: https://www.scielosp.org/pdf/rpsp/2014.v35n5$\underline{6 / 424-431 / p t}$

9. Farias LT, Sabóia RCB, Lima CHR. Obesidade e infertilidade: uma revisão sistemática da literatura. Revista Interdisciplinar da UNINOVAFAPI [serial on the internet]. 2017 [cited 2018 Nov 28];10(3):141-9. Available from: file:///D:/1326-3251-1-PB.pdf

10. Arees EA, Mayer J. Anatomical connections between medial and lateral regions of the hypothalamus concerned with food intake. Science. 2010;1967(157/796):1574-5.

11. Rodrigues LG, Pombo N, Koifman S. Prevalência de alterações metabólicas em crianças e adolescentes com sobrepeso e obesidade: uma revisão sistemática. Rev Paul Pediatr [serial on the internet]. 2011 [cited 2018 Nov 28];29(2):277-88. Available from: http:// www.scielo.br/pdf/rpp/v29n2/a21v29n2.pdf

12. Gomesa MJM, Nascimento EGC. As multifacetas do excesso de peso na criança: uma revisão sistemática. Revista de Atenção à Saúde [serial on the internet]. 2015 [cited 2018 Nov 28];13(45):70-9. Available from: http://seer.uscs.edu.br/index.php/revista ciencias saude/article/view/2667/1785

13. Mansur RB, Rizzo LB, Santos CM, Asevedo E, Cunha GR, Noto MN, et al. Adipokines, metabolic dysfunction and illness course in bipolar disorder. J Psychiatr Res. 2016;(74):63-9.

14. Souza YP, Bezerra AM, Fabrício NP, Tavares NBF, Félix NDC, Viana MCA, et al. A qualidade de vida de idosos com obesidade ou sobrepeso. Rev Bras Ciênc Saúde [serial on the internet]. 2018 [cited 2018 Nov 28];22(2):155-64. Available from: file:///D:/3517592102-1-PB.pdf

15. França BK, Alves MRM, Souto FMS, Tiziane $L$, Boaventura RF, Guimarães A, et al. Peroxidação lipídica e obesidade: métodos para aferição do estresse oxidativo em obesos. GE, J Port Gastrenterol [serial on the internet]. 2013 [cited 2018 Nov 28];20(5):199-206. Available from: https://www.sciencedirect.com/science/article/pii/ $\underline{\text { S0872817813000507 }}$

16. Souza CL, Oliveira MRM. Fatores associados ao metabolismo energético na obesidade. Nutrire. $2010 ; 35(2): 145-64$.
17. Hukshorn CJ, Platenga MS. Pegylated human recombinant leptin (PEG $O B$ ) causes additional weight loss in severely energy restricted, overweight men. Am J Clin Nutr. 2013;(77):771-6.

18. Youssef DM, Elbehidy RM, Shokry DM, Elbehidy EM. The influence of leptin on Th1/Th2 balance in obese children with asthma. J Bras Pneumol [serial on the internet]. 2013 [cited 2018 Nov 28];39(5):562-8. Available from: http://www.scielo.br/pdf/jbpneu/ v39n5/1806-3713-jbpneu-39-05-00562.pdf

19. Chaves L, Navarro AC. Compulsão alimentar, obesidade e emagrecimento. Revista Brasileira de Obesidade, Nutrição e Emagrecimento [serial on the internet]. 2011 [cited 2018 Nov 28];5(27):110-120. Available from: file:///D:/224-895-1-PB.pdf

20. Freitas MC, Ceschini FL, Ramallo BT. Resistência à insulina associada à obesidade: efeitos antiinflamatórios do exercício físico. Rev Bras Ciênc Mov [serial on the internet]. 2014 [cited 2018 Nov 28];22(3):139-47. Available from: https:// portalrevistas.ucb.br/index.php/RBCM/article/ view/4769/3381

21. Castillo-Hernandez J, Maldonado-Cervantes MI, Reyes JP, Patino-Marin N, Maldonado-Cervantes $E$, Solorzano-Rodriguez $C$, et al. A obesidade é um determinante da resistência à insulina mais importante do que os niveis circulantes de citocinas pró-inflamatórias em pacientes com artrite reumatoide. Rev Bras Reumatol [serial on the internet]. 2017 [cited 2018 Nov 28];57(4):3209. Available from: http://www.scielo.br/pdf/rbr/ v57n4/pt 0482-5004-rbr-57-04-0320.pdf

22. Velloso LA, Schwartz MW. Altered hypothalamic function in dietinduced obesity. Int $\mathrm{J}$ 0bes. 2011;35(12):1455-65.

23. Costa MC, Brito LL, Barbosa PJB, Lessa I. Adiponectina e baixo risco cardiometabólico em obesas. Arq Bras Endocrinol Metab [serial on the internet]. 2011 [cited 2018 Nov 28];55(2):146-54. Available from: http://www.scielo.br/pdf/abem/ v55n2/a07v55n2.pdf

24. Christmann AC, Zanelatto C, Semchechem CC, Novello D, Schiessel DL. Perfil de risco de doenças cardiovasculares e estado nutricional de idosos ativos de Guarapuava - Paraná. UNOPAR Cient Ciênc Biol Saúde [serial on the internet]. 2013 [cited 2018 Nov 28];15(Spec):349-56. Available from: http:// www.pgsskroton.com.br/seer/index.php/JHealthSci/ article/viewFile/570/538

25. Silva LR, Stefanello JMF, Pizzi J, Timossi LS, Leite N. Aterosclerose subclínica e marcadores inflamatórios em crianças e adolescentes obesos e não obesos. Rev Bras Epidemiol [serial on the internet]. 2012 [cited 2018 Nov 28];15(4):804-16. Available from: http://www.scielo.br/pdf/rbepid/ v15n4/12.pdf 
26. Pizzi J, Silva LR, Moser D, Leite N. Relação entre aterosclerose subclínica, pressão arterial e perfil lipídico em crianças e adolescentes obesos: uma revisão sistemática. Arq Bras Endocrinol Metab [serial on the internet]. 2013 [cited 2018 Nov 28];57(1):[about 6 pages]. Available from: http:// www.scielo.br/pdf/abem/v57n1/a01v57n1.pdf

27. Camilo DF, Ribeiro JD, Toro ADC, Baracat ECE, Barros Filho AA. Obesity and asthma: association or coincidence? J Pediatr (Rio J) [serial on the internet]. 2010 [cited 2018 Nov 28];86(1):6-14. Available from: http://www.scielo.br/pdf/jped/ v86n1/en v86n1a03.pdf

28. Brandalize $M$, Leite $N$. Alterações ortopédicas em crianças e adolescentes obesos. Fisioter Mov [serial on the internet]. 2010 [cited 2018 Nov 28];23(2):283-8. Available from: http://www.scielo. $\mathrm{br} / \mathrm{pdf} / \mathrm{fm} / \mathrm{v} 23 \mathrm{n} 2 / 11 . \mathrm{pdf}$

29. Abd El-Aziz TA, Mohamed RH, Pasha HF. Leptin, leptin gene and leptin receptor gene polymorphism in heart failure with preserved ejection fraction. Heart Vessels. 2012;(27):271-9.

30. Perfeito RR, Ribeiro PHV, Palucci MMH, Bessa MM, Santos MR. Obesidade e estresse entre trabalhadores de diversos setores de produção: uma revisão integrativa. Acta Paul Enferm [serial on the internet]. 2011 [cited 2018 Nov 28];24(4):57781. Available from: http://www.scielo.br/pdf/ape/ v24n4/a20v24n4.pdf
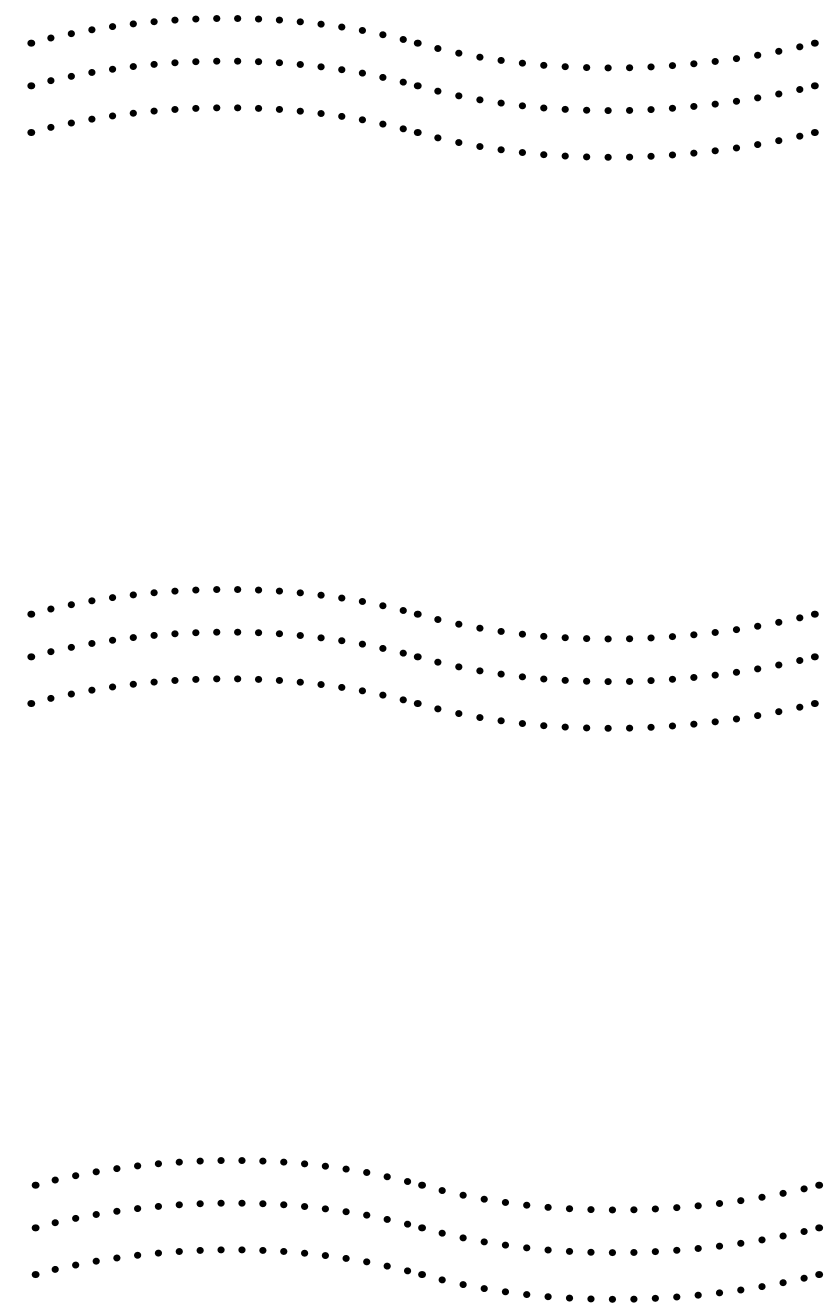
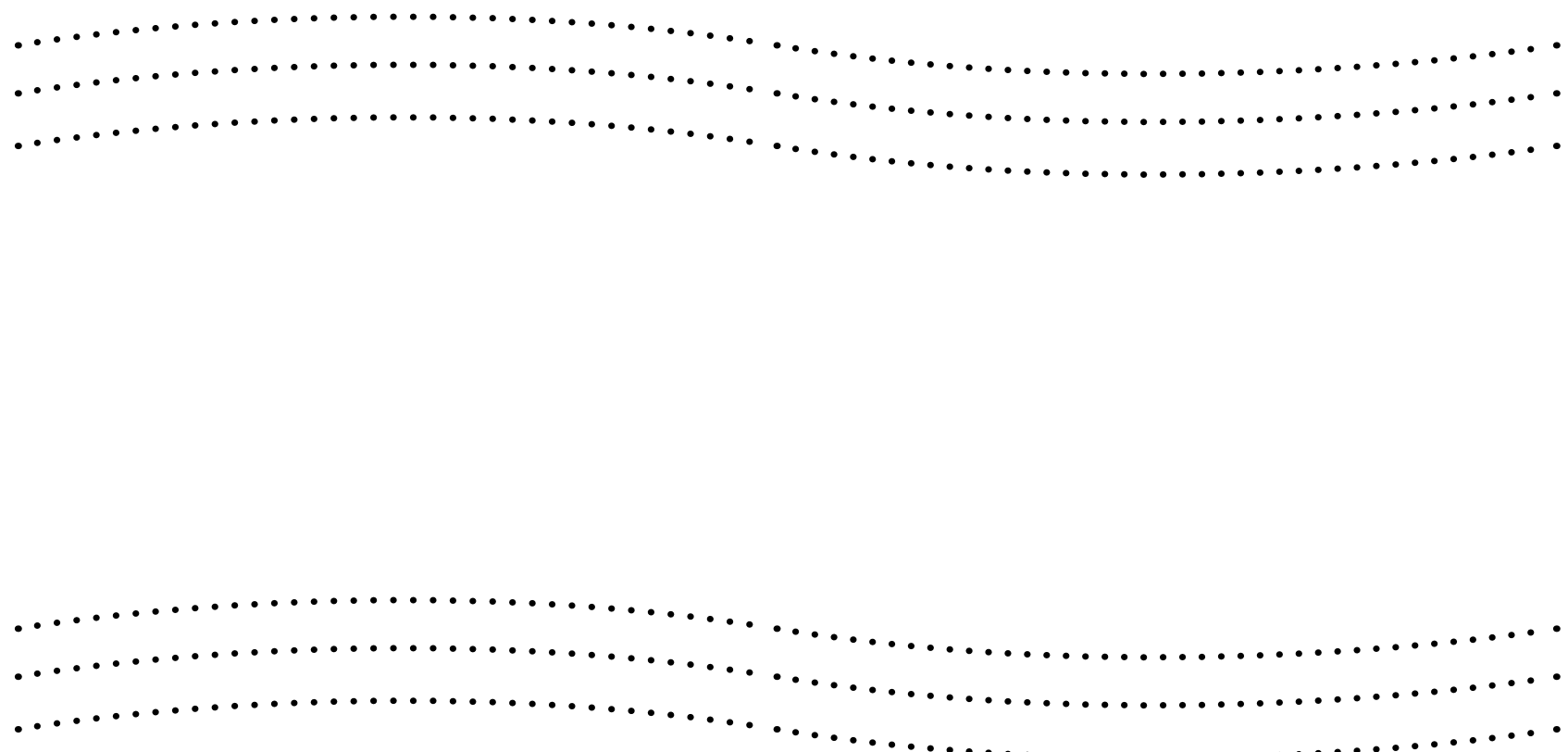